\title{
The STRATOB study: design of a randomized controlled clinical trial of Cognitive Behavioral Therapy and Brief Strategic Therapy with telecare in patients with obesity and binge-eating disorder referred to residential nutritional rehabilitation
}

\author{
Gianluca Castelnuovo ${ }^{1,2^{*}}$, Gian Mauro Manzoni ${ }^{1,3}$, Valentina Villa', Gian Luca Cesa', Giada Pietrabissa ${ }^{1,2}$ and
} Enrico Molinari ${ }^{1,2}$

\begin{abstract}
Background: Overweight and obesity are linked with Binge Eating Disorder (BED). Effective interventions to significantly reduce weight, maintain weight loss and manage associated pathologies like BED are tipically combined treatment options (dietetic, nutritional, physical, behavioral, cognitive-behavioral, pharmacological, surgical). Significant difficulties with regard to availability, costs, treatment adherence and long-term efficacy are present. Particularly Cognitive Behavioral Therapy (CBT) is the therapeutic approach indicated both in in-patient and in out-patient settings for BED. In recent years systemic and systemic-strategic psychotherapies have been implemented to treat patients with obesity and BED involved in familiar problems. Particularly a brief protocol for the systemic-strategic treatment of $\mathrm{BED}$, using overall the strategic dialogue, has been recently developed. Moreover telemedicine, a new promising low cost method, has been used for obesity with BED in out-patient settings in order to avoid relapse after the in-patient step of treatment and to keep on a continuity of care with the involvement of the same clinical in-patient team.
\end{abstract}

Methods: The comparison between CBT and Brief Strategic Therapy (BST) will be assessed in a two-arm randomized controlled clinical trial. Due to the novelty of the application of BST in BED treatment (no other RCTs including BST have been carried out), a pilot study will be carried out before conducting a large scale randomized controlled clinical trial (RCT). Both CBT and BST group will follow an in-hospital treatment (diet, physical activity, dietitian counseling, 8 psychological sessions) plus 8 out-patient telephone-based sessions of psychological support and monitoring with the same in-patient psychotherapists. Primary outcome measure of the randomized trial will be the change in the Global Index of the Outcome Questionnaire (OQ-45.2). Secondary outcome measures will be the percentage of BED patients remitted considering the number of weekly binge episodes and the weight loss. Data will be collected at baseline, at discharge from the hospital (c.a. 1 month after) and after 6-12-24 months from the end of the in-hospital treatment. Data at follow-up time points will be collected through tele-sessions.

Discussion: The STRATOB (Systemic and STRATegic psychotherapy for OBesity), a comprehensive two-phase stepped down program enhanced by telepsychology for the medium-term treatment of obese people with BED seeking intervention for weight loss, will shed light about the comparison of the effectiveness of the BST with the gold standard CBT and about the continuity of care at home using a low-level of telecare (mobile phones).

Trial registration: ClinicalTrials.gov Identifier: NCT01096251

\footnotetext{
* Correspondence: gianluca.castelnuovo@auxologico.it

'Istituto Auxologico Italiano IRCCS, Psychology Research Laboratory,

Ospedale San Giuseppe, Verbania, Italy

Full list of author information is available at the end of the article
} 


\section{Background}

One of the most important medical and public health problems of our time is represented by obesity [1]. It is widely considered as a chronic pathology and a risk factor for many medical complications such as endocrinological, pneumological, cardiovascular, and orthopedic diseases [2-8]. Overweight and obesity are also traditionally connected with Binge Eating Disorder (BED), psychopathology characterized by frequent and persistent episodes of binge eating with the key feeling of losing the control and the significative distress due to the absence of regular compensatory behaviors such as in the patients with bulimia [9]. Effective interventions to significantly reduce weight, maintain weight loss and reduce related pathologies like BED are tipically combined treatment options (dietetic, nutritional, physical, behavioral, cognitive-behavioral, pharmacological, surgical). Significant difficulties in these kinds of approaches have been underlined with regard to availability, costs, treatment adherence and long-term efficacy [10]. Even if the combined treatment options are the gold standard in weight loss, most overweight and obese individuals regain about one third of the weight lost with treatment within 1 year [11] and they will typically come back to baseline in 3 to 5 years [12-14].

About the psychological treatment of BED, Cognitive Behavioral Therapy (CBT) and Interpersonal Psychotherapy (IPT) are the psychotherapies more indicated in the Eating Disorder field [9]. Particularly CBT is the therapeutic approach indicated both in in-patient and in outpatient settings for BED [15-27].

The prevalence and spread of CBT is not under discussion, in recent years systemic, strategic and systemic-strategic psychotherapies have been implemented to treat patients with obesity and BED, overall when the pathology includes familiar characteristics and problems [26-30]. Particularly a brief protocol for the systemic-strategic treatment of BED, using overall the typical brief techniques such as the strategic dialogue, has been recently developed by Nardone and Portelli in the Handbook of Brief Strategic Therapy (BST) [31] and other contributions [32,33].

In order to deal with problems of weight regain and treatment relapses after 12-24-36 months, telemedicine, a new promising low cost method, has been used for obesity with BED in out-patient settings. This enhances the treatment after in-patients rehabilitation and keeps continuity of care through the involvement of the same clinical in-patient team [34-37].

Taking into account this scenario, we developed the STRATOB study (Systemic and STRATegic psychotherapy for OBesity), a comprehensive two-phase stepped down program enhanced by telepsychology for the medium-term treatment of obese people with BED seeking intervention for weight loss. The key aspects of the STRATOB study are the hospital-based intensive treatment and the continuity of care at home using a lowcost level of telecare (mobile phones). Many treatments delivered using new technologies, such as web-sites, e-mails, chat lines, videoconferences, UMTS mobilephones and traditional telephones could represent a valid integration for traditional psychotherapy reducing expensive and time-consuming clinical visits and improving adherence to prescribed psychological, dietetic and medical treatments through extended monitoring and support [10,12,34,38-46].

This paper describes the design of the STRATOB study, a small scale two-arm randomized controlled clinical trial (RCT). The main aim of this study will be to compare the effectiveness of the BST [31-33] with the gold standard CBT [26,47-53] in a in-patient and telephone-based out-patient program for a sample of obese people with BED seeking treatment for weight reduction.

\section{Methods}

The comparison between the CBT and BST will be assessed in a two-arm randomized controlled clinical trial. Participants will be randomly allocated in 2 groups:

1) CBT group: in-hospital treatment (diet, physical activity, dietitian counseling, 8 sessions of CBT) plus 8 out-patient telephone-based sessions of CBT-oriented psychological support and monitoring with the same CBT in-patient psychotherapists;

2) BST group: in-hospital treatment (diet, physical activity, dietitian counseling, 8 sessions of BST) plus 8 out-patient telephone-based sessions of BST-oriented psychological support and monitoring with the same BST in-patient psychotherapists;

The Medical Ethics Committee of Istituto Auxologico Italiano approved the study protocol and the Informed Consents.

The rehabilitation program considered in this study will have a total duration of 7 months and consists of two stepped down phases: in-patient (1 month) and outpatient (the following 6 months).

During the in-patient phase, participants will undergo an intensive four-week hospital-based and medicallymanaged rehabilitation program for weight reduction. Along this period, participants will live in a hospital located on a mountain highland and far away from towns and cities. Visits from family members and friends are allowed only in the afternoon. All patients will be placed on a hypocaloric nutritionally balanced diet tailored to the individual after consultation with a dietitian (energy intake around $80 \%$ of the basal energy expenditure estimated according to the Harris-Benedict equation and a macronutrient composition of about $16 \%$ proteins, $25 \%$ 
fat and 59\% carbohydrates). Furthermore, they will receive nutritional counseling provided by dietitians, psychotherapy provided by psychologists-psychotherapists (well trained in CBT and BST approaches) and have physical activity training provided by physiotherapists.

Nutritional rehabilitation program aims to improve and promote change in eating habits and consists of both individual sessions (dietary assessment, evaluation of nutrient intake and adequacy, nutritional and anthropometric status, eating patterns, history of overweight, readiness to adopt change) and group sessions (45 minutes each session, twice a week, including: information on obesity and related health risks, setting of realistic goals for weight loss, healthy eating in general, general nutrition and core food groups, weight management and behavior change strategies for preventing relapse).

Psychotherapy will be provided twice a week in individual setting. CBT individual sessions, lasting 45 minutes each, are mainly based on the cognitive-behavioral approach described by Cooper and Fairburn [53] and emphasize the techniques of self-monitoring, goal setting, time management, prompting and cueing, problem solving, cognitive restructuring, stress management and relapse prevention. BST individual sessions, lasting 45 minutes each, are mainly based on the brief strategic approach described by Nardone and colleagues [31-33] and emphasize the techniques of working on "attempted solutions" (such as keeping control by abstaining from food and continuously striving to exert self-control with a subsequent loss of control), using reframing maneuvers, inducing fear of fasting rather then bingeing, understanding what is maintaining and worsening the problem, etc. (p. 105-105, [31]).

Physical activity will take place once a day except for week-end and consists of group programs (20 subjects) based on postural gymnastics, aerobic activity and walks in the open. In-patients with specific orthopedic complications will carry out individual activities planned by physiotherapists and articulated in programs of physical therapy, assisted passive and active mobilization and isokinetic exercise.

At the end of the in-patient step low to moderate weight losses will be expected and it is important to underline that weight loss is not the primary goal of the in-patient program (each patient is made clear about this point at the very beginning of the treatment). Beyond the medical management of metabolic risk factors for health such as type 2 diabetes, developing a sense of autonomy and competence will be the primary purposes of the inhospital interventions. Skills and tools for change will be provided to patients and they will be supported in assigning positive values to healthy behaviors and also in aligning them with personal values and lifestyle patterns.
Just after discharge, participants will have 4 telephone calls with their in-patient psychotherapists along 2 months. From the 3rd to the 6th month sessions will be scheduled every 30 days. During tele-sessions, psychotherapists will test the out-patients' progress, their mood, the maintenance of functional alimentary and physical activity habits, the loss/increase of weight and will discuss about critical moments. Some research indicates that changes in behavior (eating and exercise) often follow discrete moments which have been variably described as life events or life crises [54]. Life events can lead to weight loss but also to weight gain and qualitative research shows that it is not the event per se that results in behavior change but the ways in which this event is appraised, interpreted and managed by the individual [55]. Psychotherapists (both CBT and BST) will have thus the opportunity to discuss with their patients in remote settings about the significant events during the telephone calls and will cognitively reconstruct dysfunctional appraisals in functional ways. In particular, phonesessions with the psychotherapists will aim to consolidate strategies and abilities acquired during the in-patient phase, to improve self-esteem and self-efficacy, to support motivation, to prevent relapse and to provide problem-solving and crisis counseling.

As underlined, in the out-patient step of the STRATOB program great relevance will be given to the clinician-patient relationship as an important medium and vehicle of change [56]. After discharge, out-patients will begin to experience the autonomy and competence to change they will have developed during the in-patient phase and inevitably face resistances and barriers. Outpatients will be supported by telephone by the psychotherapists who will have attended them during the in-hospital phase in exploring resistances and barriers they experience and in finding functional pathways to cope. Furthermore, out-patients will be helped to experience mastery in terms of the health behavior change that needs to be engaged.

\section{Recruitment of the study population}

One hundred consecutive female in-patients with obesity and BED in the age range of 18-65 years, who will be referred to a single clinical center (Saint Joseph Hospital - Istituto Auxologico Italiano IRCCS) for weightloss treatment, will be asked and screened for admission to the study. Patients will be sequentially recruited.

\section{Inclusion and exclusion criteria}

In-patients will be eligible when they will meet the following inclusion criteria at the admission to the hospital: 1) age between 18 and 65 years; 2) obesity according to the WHO criteria (BMI $\geq 30$ ); 3) BED (DSM-IV-TR 
criteria) and 4) written and informed consent to participate. Exclusion criteria for the study are: 1) other severe psychiatric disturbance diagnosed by DSM-IV-TR criteria; 2) concurrent medical condition not related to obesity. SCID (Structured Clinical Interview for DSM-IV-TR Disorders) I and II [57] will be used as screening tools for psychiatric disorders and will be administered by an independent clinical psychologist as part of his work.

\section{Randomization procedure}

All participants will be randomly assigned to the CBT or BST group. The randomization scheme will be generated using the Web site Randomization.com (http:// www.randomization.com). Randomization will occur after the baseline measurements.

\section{Patient Assessment and Measurements}

Primary outcome measure of the randomized trial will be the change in the Global Index of the Outcome Questionnaire (OQ 45.2).

Secondary outcome measures will be the percentage of BED patients remitted considering the number of weekly binge episodes and the weight loss. Data will be collected at baseline, at discharge from the hospital (c.a. 1 month after) and after 6-12-24 months from the end of in-hospital treatment.

Weight will be assessed with the participant in lightweight clothing with shoes removed on a balance beam scale.

Data collected at follow-up time points will be collected through tele-sessions.

\section{Psychological and behavioral assessment}

Participants will complete the Outcome Questionnaire (OQ 45.2) - Italian translation and validation [58,59] at entry to the study and at discharge from the hospital with a self-report procedure. The number of weekly binge episodes will be assessed with a self-report procedure at entry to the study and at discharge from the hospital. At 6-12-24 month follow-up data will be collected via telephone during the tele-sessions.

\section{Outcome Questionnaire (OQ 45.2)}

The OQ 45.2 is a self report questionnaire developed by Michael Lambert in 1996 [60]. The OQ 45 items version is a measure of outcome and it is designed in order to collect repeated measures of patient progress during psychotherapy and after its conclusion. This instrument is one of the most used in psychotherapy research in the U.S. [61]. The OQ 45.2 is composed by 45 items that form 3 scales: Symptom Distress (SD), Interpersonal Relations (IR) e Social Role (SR), and a Global Index. The OQ 45.2 is proposed as a brief screening and outcome scale that tries to assess the subjective experience of a person, as well as the way he or she functions in the world [60]. The choice of this questionnaire has to take into account the need to measure a change in a short time (1 month of in-patient treatment) due to psychotherapeutic activities. The Italian version of the OQ-45.2 appears promising as a measure of general psychological distress, and it could be used to measure the psychotherapy outcome in routine clinical practice. So the Outcome Questionnaire- 45 was developed as a brief measure of client progress in psychotherapy to address the need for accountability in the era of managed care and to improve upon extant outcome measures.

The investigator involved in administering and interpreting the OQ45.2 will be blinded to treatment assignment.

\section{Psychotherapists and Treatment Fidelity}

Treatments will be delivered by 4 experienced and certified psychotherapists from diverse backgrounds with specific training in CBT and BST. Psychotherapists will receive monthly supervision by senior certified psychotherapists to ensure competent and uniform treatment delivery.

\section{Sample size calculation and statistical analysis}

Due to the novelty of the application of BST in BED treatment, no other RCTs including BST have been carried out. So this RCT will be similar to a pilot study, typically defined as small scale preliminary study, and according to Lackey and Wingate [62], a pilot work may use at least the $10 \%$ of the sample required. Using an Apriori Sample Size Calculator for Student's t-Test (G*Power 3.1.2 software), 394 participants per group will be needed to detect a difference with an estimated Effect Size (Cohen's d) of 0.2, an alpha of 0.05 two-sided, a desired statistical power of 0.80 . We decided not to make assumptions on sidedness not having previous results about comparison between CBT and BST. Taking into account the need of at least $10 \%$ of the sample required (788 individuals, $\mathrm{n}=394$ per group), 80 persons $(\mathrm{n}=40$ per group) will be considered enough.

Weight data will be analyzed with an intention-totreat (ITT) approach with dropouts assumed to have regained $0,3 \mathrm{~kg}$ per month, an assumption already used in previous studies $[12,63]$. Differently, missing data in the other variables will be replaced with baseline observation carried forward (BOCF) or last observation carried forward (LOCF) as appropriate, assuming no improvement for non-responders patients.

The independent samples $t$ test will be used to examine between-group differences in $\mathrm{OQ}$ variables and weight at all time-points. Change scores will be also examined. Chisquare will be used to test the association between treatment groups and number of weekly binge episodes $\geq 2$ at 
6 months. Corrected effect sizes (Hedges) and 95\% Confidence Interval will be calculated for both between-group and with-in group differences.

All data analyses will be performed using the Statistical Package for the Social Sciences (version 16.0; SPSS, Inc., Chicago, IL).

\section{Discussion}

Due to the novelty of Brief Strategic Therapy (BST) in the treatment of Binge Eating Disorders, no well defined hypothesis have been established about the possible contribution of this kind of therapy in comparison with Cognitive Behavior Therapy (CBT), the "gold standard" psychological treatment for BED. With the development of a brief systemic-strategic clinical protocol for treatment of BED [31-33], new interventions in the field of eating disorders are available and have to be evaluated. Systemic and systemic-strategic psychotherapies such as BST traditionally focus on systemic-family problems and contexts and these areas of interventions could represent a real added value in comparison with CBT. Moreover, BST sessions could emphasize the techniques of working on "attempted solutions", using reframing maneuvers, inducing fear of fasting rather then bingeing, understanding what is maintaining and worsening the problem, etc. (p. 105-106, [31]). This focus on "attempted solutions", that in the typical BED are related to dysfunctional habits such as "trying to keep control by abstaining from food and continuously striving to exert self-control with a subsequent loss of control", could generate some differences between the BST and the CBT approach about the remission of weekly binge episodes at 6-12-24 months (during the in-patient phase patients will do not cope with the binge temptations due to the lack of them in a safe place like the hospital). Moreover the focus of BST on symptoms reduction (in particular anxiety and depression) in short time (traditionally 6-12 months) could generate significant difference between CBT and BST in the OQ 45.2 Symptom Distress (SD) scale.

The real therapeutic impact of CBT and BST could be significantly reduced by the use of a non-traditional setting in the second part of the trial. Even if telecare could represent a great opportunity for the continuity of treatments "moving the healthcare where it really needs", the limitations of non-verbal communications, due to the use of telephone sessions, could influence the $\mathrm{CBT}$ and BST protocols in different ways that have to be investigated.

A possible limitation in this trial is the small sample size. Even if this is the first study that compared CBT with BST in the treatment of BED, larger clinical trials will be needed in order to evaluate both the effectiveness of integrated psychotherapies (such as the traditional CBT combined with BST or IPT) and the use of new technologies in the clinical field (from the "low technology" telephone to the "medium technology" clinically oriented SMS, MMS, e-mail, chat, web sources, till the "high technology" web 2.0 or virtual and augmented realities) [64-70].

Moreover generalization of research findings will be limited because the STRATOB trial will be a single-centre randomized controlled study. Future multi-centre trials should extend both the use of alternative models of psychological support or psychotherapy, i.e. BST, and the implementation of new technologies, in order to find the best combination of the variables involved (psychotherapeutic model, level of technology, number of sessions, etc.).

Taking into account that traditional interventions for BED patients with obesity typically fails because of low compliance with clinical programs, continuous monitoring and psychological support are needed. This study will improve the evidence-based knowledge of how CBT and BST may enhance the long-term efficacy of clinical interventions for $B E D$ and weight loss using telecare settings too.

Due to the growing development of technologies in the clinical applications, we are moving towards a new "health care everywhere" approach, where clinicians could provide their treatments in both traditional clinical settings (public and private hospitals, clinics, services, etc.) and innovative clinical settings (remote outpatients' clinics, tele-health and e-health based settings) $[65,68,70-72]$.

\section{List of abbreviations used}

BED: Binge Eating Disorder; CBT: Cognitive Behavioral Therapy; IPT: Interpersonal Psychotherapy; BST: Brief Strategic Therapy; OQ: Outcome Questionnaire; RCT: Randomized Clinical Trial; STRATOB: Systemic and STRATegic psychotherapy for OBesity; SMS: Short Message Service; MMS: Multimedia Messaging Service; SD: Symptom Distress; IR: Interpersonal Relations; SR: Social Role; UMTS: Universal Mobile Telecommunications System; SCID: Structured Clinical Interview for DSM Disorders; DSM: Diagnostic and Statistical Manual of Mental Disorders.

\section{Acknowledgements}

The STRATOB project is a preliminary low technology-based part of the more comprehensive high-technology based TECNOB Project supported by the "Compagnia di San Paolo" private foundation. No technological partners are involved in this STRATOB project.

\section{Author details}

'Istituto Auxologico Italiano IRCCS, Psychology Research Laboratory, Ospedale San Giuseppe, Verbania, Italy. ${ }^{2}$ Department of Psychology, Catholic University of Milan, Italy. ${ }^{3}$ Department of Psychology, University of Bergamo, Italy.

\section{Authors' contributions}

GC conceived the study, planned its design and made substantial contribution to the manuscript drafting. GMM participated in the study design and contributed to the manuscript drafting. W, GLC, GP and EM participated in the study design and helped to draft the manuscript. All authors read and approved the final manuscript.

\section{Competing interests}

The authors declare that they have no competing interests. 
Received: 21 March 2011 Accepted: 9 May 2011 Published: 9 May 2011

\section{References}

1. WHO: Definition and Diagnosis of Diabetes Mellitus and Intermediate Hyperglycemia. Report of a WHO/IDF consultation 2006

2. Pi-Sunyer X: The medical risks of obesity. Postgrad Med 2009, 121:21-33.

3. Gnacinska M, Malgorzewicz S, Stojek M, Lysiak-Szydlowska W, Sworczak K: Role of adipokines in complications related to obesity: a review. Adv Med Sci 2009, 54:150-157.

4. van Kruijsdijk RC, van der Wall E, Visseren FL: Obesity and cancer: the role of dysfunctional adipose tissue. Cancer Epidemiol Biomarkers Prev 2009, 18:2569-2578.

5. McCallister JW, Adkins EJ, O'Brien JM Jr: Obesity and acute lung injury. Clin Chest Med 2009, 30:495-508, viii.

6. Shah N, Roux F: The relationship of obesity and obstructive sleep apnea. Clin Chest Med 2009, 30:455-465, vii.

7. Beleigoli AM, Diniz MF, Ribeiro AL: Natriuretic peptides: linking heart and adipose tissue in obesity and related conditions-a systematic review. Obes Rev 2009, 10:617-626.

8. Momeni A, Heier M, Bannasch H, Stark GB: Complications in abdominoplasty: a risk factor analysis. J Plast Reconstr Aesthet Surg 2009, 62:1250-1254.

9. Wilson GT, Wilfley DE, Agras WS, Bryson SW: Psychological treatments of binge eating disorder. Arch Gen Psychiatry 67:94-101.

10. Weinstein PK: A review of weight loss programs delivered via the Internet. J Cardiovasc Nurs 2006, 21:251-258, quiz 259-260.

11. Hill JO, Thompson H, Wyatt H: Weight maintenance: what's missing? J Am Diet Assoc 2005, 105:S63-66.

12. Wing RR, Tate DF, Gorin AA, Raynor HA, Fava JL: A self-regulation program for maintenance of weight loss. N Engl J Med 2006, 355:1563-1571.

13. Jeffery RW, Drewnowski A, Epstein LH, Stunkard AJ, Wilson GT, Wing RR, Hill DR: Long-term maintenance of weight loss: current status. Health Psychol 2000, 19:5-16.

14. Katan MB: Weight-loss diets for the prevention and treatment of obesity. N Engl J Med 2009, 360:923-925.

15. Schlup B, Munsch S, Meyer AH, Margraf J, Wilhelm FH: The efficacy of a short version of a cognitive-behavioral treatment followed by booster sessions for binge eating disorder. Behav Res Ther 2009, 47:628-635.

16. Sarwer DB: Comment on: brief, four session group CBT reduces binge eating behaviors among bariatric surgery candidates (Ashton K, et al. 2009;5:257-62. Surg Obes Relat Dis 2009, 5:407-408.

17. Ashton K, Drerup M, Windover A, Heinberg L: Brief, four-session group CBT reduces binge eating behaviors among bariatric surgery candidates. Surg Obes Relat Dis 2009, 5:257-262.

18. Tsiros MD, Sinn N, Brennan L, Coates AM, Walkley JW, Petkov J, Howe PR, Buckley JD: Cognitive behavioral therapy improves diet and body composition in overweight and obese adolescents. Am J Clin Nutr 2008, 87:1134-1140.

19. Munsch S, Roth B, Michael T, Meyer AH, Biedert E, Roth S, Speck V, Zumsteg U, Isler E, Margraf J: Randomized controlled comparison of two cognitive behavioral therapies for obese children: mother versus motherchild cognitive behavioral therapy. Psychother Psychosom 2008, 77:235-246.

20. Shelley-Ummenhofer J, MacMillan PD: Cognitive-behavioural treatment for women who binge eat. Can J Diet Pract Res 2007, 68:139-142.

21. Eichler K, Zoller M, Steurer J, Bachmann LM: Cognitive-behavioural treatment for weight loss in primary care: a prospective study. Swiss Med Wkly 2007, 137:489-495.

22. Brownley KA, Berkman ND, Sedway JA, Lohr KN, Bulik CM: Binge eating disorder treatment: a systematic review of randomized controlled trials. Int J Eat Disord 2007, 40:337-348.

23. Vaidya V: Cognitive behavior therapy of binge eating disorder. Adv Psychosom Med 2006, 27:86-93.

24. Grilo CM, Masheb RM, Wilson GT: Rapid response to treatment for binge eating disorder. J Consult Clin Psychol 2006, 74:602-613.

25. Fennig S: Can we treat morbid obese children in a behavioral inpatient program? Pediatr Endocrinol Rev 2006, 3 Suppl 4:590-596.

26. Berkman ND, Bulik CM, Brownley KA, Lohr KN, Sedway JA, Rooks A, Gartlehner G: Management of eating disorders. Evid Rep Technol Assess (Full Rep) 2006, 1-166.

27. Wilson GT: Psychological treatment of eating disorders. Annu Rev Clin Psychol 2005, 1:439-465.
28. Peterson Y: Family therapy treatment: working with obese children and their families with small steps and realistic goals. Acta Paediatr Suppl 2005, 94:42-44

29. Molinari E, Baruffi $M$, Croci M, Marchi S, Petroni ML: Binge eating disorder in obesity: comparison of different therapeutic strategies. Eat Weight Disord 2005, 10:154-161.

30. Wilfley DE, Cohen LR: Psychological treatment of bulimia nervosa and binge eating disorder. Psychopharmacol Bull 1997, 33:437-454.

31. Nardone G, Portelli C: Knowing through changing: The Evolution of Brief Strategic Therapy Crown House Publishing Limited; 2005.

32. Milanese R: Advanced Brief therapy for eating disorders. Brief Strategic and Systemic Therapy European Review 2004, 1:125-129.

33. Nardone G, Barbieri Brook R: Advanced Brief Strategic Therapy: An overview of interventions with eating disorders to exemplify how theory and practice work. European Journal of Psychotherapy \& Counselling 2010, 12:113-127.

34. Marks S, Shaikh U, Hilty DM, Cole S: Weight status of children and adolescents in a telepsychiatry clinic. Telemed J E Health 2009, 15:970-974.

35. Shaikh U, Cole SL, Marcin JP, Nesbitt TS: Clinical management and patient outcomes among children and adolescents receiving telemedicine consultations for obesity. Telemed J E Health 2008, 14:434-440.

36. Riva G, Alcaniz M, Anolli L, Bacchetta M, Banos R, Beltrame F, Botella C, Galimberti C, Gamberini L, Gaggioli A, et al: The VEPSY UPDATED project: technical and clinical rationale. Stud Health Technol Inform 2002, 85:395-401.

37. Riva G, Alcaniz M, Anolli L, Bacchetta M, Banos R, Beltrame F, Botella C, Galimberti C, Gamberini L, Gaggioli A, et al: The VEPSY updated project: virtual reality in clinical psychology. Cyberpsychol Behav 2001, 4:449-455.

38. Tate DF, Jackvony EH, Wing RR: Effects of Internet behavioral counseling on weight loss in adults at risk for type 2 diabetes: a randomized trial. JAMA 2003, 289:1833-1836.

39. Tate DF, Jackvony EH, Wing RR: A randomized trial comparing human email counseling, computer-automated tailored counseling, and no counseling in an Internet weight loss program. Arch Intern Med 2006, 166:1620-1625.

40. Tate DF, Wing RR, Winett RA: Using Internet technology to deliver a behavioral weight loss program. JAMA 2001, 285:1172-1177.

41. Harvey-Berino J, Pintauro S, Buzzell P, Gold EC: Effect of internet support on the long-term maintenance of weight loss. Obes Res 2004, 12:320-329.

42. Haugen HA, Tran ZV, Wyatt HR, Barry MJ, Hill JO: Using telehealth to increase participation in weight maintenance programs. Obesity (Silver Spring) 2007, 15:3067-3077.

43. Shapiro JR, Bauer S, Hamer RM, Kordy H, Ward D, Bulik CM: Use of text messaging for monitoring sugar-sweetened beverages, physical activity, and screen time in children: a pilot study. J Nutr Educ Behav 2008, 40:385-391.

44. McTigue KM, Conroy MB, Hess R, Bryce CL, Fiorillo AB, Fischer GS, Milas NC, Simkin-Silverman LR: Using the internet to translate an evidence-based lifestyle intervention into practice. Telemed J E Health 2009, 15:851-858.

45. Krukowski RA, Harvey-Berino J, Ashikaga T, Thomas CS, Micco N: Internetbased weight control: the relationship between web features and weight loss. Telemed J E Health 2008, 14:775-782.

46. Schiel R, Beltschikow W, Radon S, Kramer G, Schmiedel R, Berndt RD, Stein G: Long-term treatment of obese children and adolescents using a telemedicine support programme. J Telemed Telecare 2008, 14:13-16

47. Hay PP, Bacaltchuk J, Stefano S, Kashyap P: Psychological treatments for bulimia nervosa and binging. Cochrane Database Syst Rev 2009, CD000562.

48. Hay PJ, Bacaltchuk J: Bulimia nervosa. Clin Evid (Online) 2008, 2008.

49. Hay PJ, Bacaltchuk J, Stefano S: Psychotherapy for bulimia nervosa and binging. Cochrane Database Syst Rev 2004, CD000562.

50. Hay PJ, Bacaltchuk J: Psychotherapy for bulimia nervosa and binging. Cochrane Database Syst Rev 2003, CD000562.

51. Hay PJ, Bacaltchuk J: Psychotherapy for bulimia nervosa and binging. Cochrane Database Syst Rev 2001, CD000562.

52. Hay PJ, Bacaltchuk J: Psychotherapy for bulimia nervosa and binging. Cochrane Database Syst Rev 2000, CD000562.

53. Cooper HM: Meta-analysis and the integrative research review. In Research methods in personality and social psychology. Edited by: Hendrik C, Clark MS. Newbury Park: Sage; 1990:142-163.

54. Ogden J, Hills L: Understanding sustained behavior change: the role of life crises and the process of reinvention. Health (London) 2008, 12:419-437. 
55. Ogden J, Stavrinaki M, Stubbs J: Understanding the role of life events in weight loss and weight gain. Psychol Health Med 2009, 14:239-249.

56. Ryan RM, Patrick H, Deci EL, Williams GC: Facilitating health behaviour change and its maintenance: Interventions based on Self-Determination Theory. The European Health Psychologist 2008, 10:2-5.

57. Perone R: SCID I/P DSM IV-TR Research Version. 2002

58. Chiappelli M, Lo Coco G, Gullo S, Bensi L, Prestano C: [The outcome questionnaire 45.2. Italian validation of an instrument for the assessment of psychological treatments]. Epidemiol Psichiatr Soc 2008, 17:152-161.

59. Lo Coco G, Chiappelli M, Bensi L, Gullo S, Prestano C, Lambert MJ: The factorial structure of the Outcome Questionnaire-45: a study with an Italian sample. Clin Psychol Psychother 2008, 15:418-423.

60. Lambert MJ, Hansen NB, Umphress V, Lunnen K, Okiishi J, Burlingame GM, Reisenger CW: Administration and scoring manual for the Outcome Questionnaire (OQ 45.2) Stevenson, MD: American Professional Credentialing Services LLC; 1996.

61. Hatfield DR, Ogles BM: The Use of Outcome Measures by Psychologists in Clinical Practice. Professional Psychology: Research and Practice 2004, 35:485-491.

62. Lackey NR, Wingate AL: The pilot study: one key to research success. Kans Nurse 1986, 61:6-7.

63. Brownell KD, Wadden TA: Etiology and treatment of obesity: understanding a serious, prevalent, and refractory disorder. J Consult Clin Psychol 1992, 60:505-517.

64. Riva G, Bacchetta M, Cesa G, Conti S, Castelnuovo G, Mantovani F, Molinari E: Is severe obesity a form of addiction? Rationale, clinical approach, and controlled clinical trial. Cyberpsychol Behav 2006, 9:457-479.

65. Castelnuovo G, Buselli C, De Ferrari R, Gaggioli A, Mantovani F, Molinari E, Villamira M, Riva G: New tools in cybertherapy: the VEPSY web site. Stud Health Technol Inform 2004, 99:15-35.

66. Riva G, Botella C, Castelnuovo G, Gaggioli A, Mantovani F, Molinari E: Cybertherapy in practice: the VEPSY updated project. Stud Health Technol Inform 2004, 99:3-14

67. Riva G, Alcaniz M, Anolli L, Bacchetta M, Banos R, Buselli C, Beltrame F, Botella C, Castelnuovo G, Cesa G, et al: The VEPSY UPDATED Project: clinical rationale and technical approach. Cyberpsychol Behav 2003, 6:433-439.

68. Castelnuovo G, Gaggioli A, Mantovani F, Riva G: From psychotherapy to etherapy: the integration of traditional techniques and new communication tools in clinical settings. Cyberpsychol Behav 2003, 6:375-382.

69. Gaggioli A, Mantovani F, Castelnuovo G, Wiederhold B, Riva G: Avatars in clinical psychology: a framework for the clinical use of virtual humans. Cyberpsychol Behav 2003, 6:117-125.

70. Castelnuovo G: No medicine without psychology: the key role of psychological contribution in clinical settings. Frontiers in Psychology for Clinical Settings 2010, 1.

71. Castelnuovo G, Manzoni GM, Cuzziol P, Cesa GL, Tuzzi C, Villa V, Liuzzi A Petroni ML, Molinari E: TECNOB: study design of a randomized controlled trial of a multidisciplinary telecare intervention for obese patients with type-2 diabetes. BMC Public Health 10:204.

72. Castelnuovo G: Empirically supported treatments in psychotherapy: towards an evidence-based or evidence-biased psychology in clinical settings. Front Psychology 2010, 1:27.

doi:10.1186/1745-6215-12-114

Cite this article as: Castelnuovo et al.: The STRATOB study: design of a randomized controlled clinical trial of Cognitive Behavioral Therapy and Brief Strategic Therapy with telecare in patients with obesity and bingeeating disorder referred to residential nutritional rehabilitation. Trials 2011 12:114.

\section{Submit your next manuscript to BioMed Central and take full advantage of:}

- Convenient online submission

- Thorough peer review

- No space constraints or color figure charges

- Immediate publication on acceptance

- Inclusion in PubMed, CAS, Scopus and Google Scholar

- Research which is freely available for redistribution

Submit your manuscript at www.biomedcentral.com/submit
Ciomed Central 